\title{
Extending the ODMG Standard with Views
}

\author{
Jesús García-Molina, María-José Ortín-Ibáñez, Ginés García-Mateos \\ Departamento de Informática y Sistemas. Universidad de Murcia \\ 30071 Campus de Espinardo, Murcia, Spain \\ \{jmolina, mjortin, ginesgm \}@um.es
}

\begin{abstract}
Views are an important functionality provided by the relational database systems. However, commercial object-oriented database systems do not support a view mechanism because defining the semantics of views in the context of an object-oriented model is more difficult than in the relational model. Indeed, views are not included in the ODMG standard. In this paper, we present a proposal aimed at including views in the ODMG, by extending the object model and the object definition language (ODL). We consider object-oriented views as having the same functionality as relational views. Views are included in the object model in such a way that i) views are a new kind of data type definition, just as are classes, interfaces and literals, ii) an IS-VIEW relationship is introduced in order to specify the derivation of a view from its base class, and iii) a view instance preserves the identity of its base instance. A view can import attributes, relationships and operations from its base class, and it can also add new operations, derived attributes and derived relationships. The extent of the view is defined by an object query language (OQL) predicate. We also describe a $\mathrm{C}++$ binding showing the practicability of the proposed model.
\end{abstract}

Keywords. View model. View management. Object-oriented database system. ODMG standard.

\section{Introduction}

Since the object-oriented $(\mathrm{OO})$ data model is more complex than the relational model, commercial object-oriented database management systems (OODBMS) do not provide some of the important functionalities supported by relational database systems. This fact has contributed to OODBMS not achieving the success expected [4]. Views are an example of such functionalities which have not been made available in OODBMS. In the past decade, a lot of effort has been devoted to defining the view concept for OO data model and several OO view models have been proposed [1,6,7-11]. However, despite this research, today the commercial systems do not support views and the ODMG standard [5] does not include views, although they are recognized as an indispensable functionality in OODBMS.

In this paper, we present a proposal to include views in the ODMG and in such a way that they preserve the same functionality as relational views. The inclusion of views in the standard requires extending its components: the object model, the object definition language (ODL) and the binding to the programming languages. In our proposal, views are a new kind of data type added to the types already existing in the ODMG object model: classes, interfaces and literals. Views are types which are defined by an $I S$-VIEW relationship applied to a base class (or view). The instances of a view preserve the identity of the instances of the base class and thus a view does not generate new instances. The definition of a view is made up of three components: i) list of features (attributes, relationships and operations) imported from its base class, ii) list of additional features (operations, derived attributes and derived relationships) and iii) an object query language (OQL) predicate that determines the extent of the view. Any proposal for extending the ODMG has to show how the extensions are mapped into the programming languages. In our case, the proposed view model has been 
mapped into the $\mathrm{C}++$ language.

Other proposals for extending the ODMG standard have been published. In [2,3] the ODMG object model is extended with time and composite objects respectively. To our knowledge, the object-oriented view models proposed to date have not been defined in the context of the ODMG standard. Moreover, little work has been done with regard to the implementation aspects of $\mathrm{OO}$ view models. Therefore, the contribution of our work is twofold: we have defined a view model for the ODMG and we have dealt with implementation issues by defining a $\mathrm{C}++$ binding which shows the practicability of the proposed view model.

The rest of this paper is organized as follows. In Section 2, we present our main design choices. Next, we summarize the basic concepts of the ODMG object model. In section 4, we describe the features of the proposed view model by showing how to extend the ODMG object model and the ODL to include the definition of views. In Section 5, we explain the binding between ODL specifications including views and $\mathrm{C}++$. In Section 6, we describe how to access the objects stored in the database through views. Finally, in Section 7, we present our conclusions and future work is outlined.

\section{Main Design Choices}

Throughout this paper we use the following terminology. The term base class denotes the class (or view) from which a view is derived. The global database schema contains all the classes, whereas an external schema contains a set of views and a set of classes imported from the global schema. We distinguish between stored objects (instances of a class) and view instances (instances of a base class which also belong to the view).

As is indicated in [6,7], the two main dimensions in the design of an $\mathrm{OO}$ view mechanism are the placement of views in the database schema (a class hierarchy), and the nature of view instances. The design choices in each dimension are influenced by the data model and the view functionality chosen. Obviously, our data model is the ODMG object model. With regard to the view functionality, we consider it appropriate that $\mathrm{OO}$ views can be used for the same purposes as relational views, that is: i) definition of external schemas (data independence), ii) content-based authorizations (data protection), and iii) shorthand for queries.

Some proposals have explored the applicability of views to the simulation of database schema evolution $[7,8,9,11]$. However, as is pointed out in [8], we think that only a range of changes to a database schema should be simulated by views. Since a view is a mechanism for deriving data from the stored database, the simulation of changes involving database restructuring -such as the definition of new attributes not derived from existing data-complicates the view model excessively. These modifications are expensive and make the dynamic addition and removal of views very difficult, whereas such operations are common in relational systems. We think that a view model should preserve the inde- 
pendence between the conceptual schema and the view mechanism, that is, the structure of objects stored in the database should not be modified when new views are defined.

A view mechanism supporting the definition of external schemas upon which it is possible to develop OO applications, requires OO views to have the same nature as classes and interfaces, just as a relational view can be used as a table. Therefore, a view should be a new kind of data type. In this way, a view can be used in any context on which a class or interface may appear, for example, if static typed is assumed, then the type of a program entity (attribute, parameter or local variable) could be a view.

\subsection{Placement of Views in the Database Schema}

Since a view is a data type and the database schema is a type hierarchy, when solving the view placement problem the semantic of the subtype/supertype inheritance-based relationships has to be kept in mind. A detailed discussion about possible solutions is presented in [7], where a new view hierarchy, separated from the class hierarchy, is put forward as the most appropriate solution; each view is connected to its base class by the view derivation relationship defined in [1] and a view inheritance relationship, similar to the class inheritance relationship, is introduced. This approach has also been considered in other models $[8,11]$. In our proposal, we introduce both the view derivation relationship and the view inheritance relationship in the ODMG object but only one single class hierarchy is obtained in the $\mathrm{C}++$ binding and there is no separated hierarchy for views.

\subsection{Nature of the View Instances}

As is indicated in [7,10], two kinds of views can be identified: object-preserving views, whose instances have the same object identifier as the base instances from which they have been generated, and object-generating views, which create new instances, generating new object identifiers. Object-preserving views facilitate the implementation of the view mechanism and the convenience of using them is described in [10], where it is indicated that object preservation is crucial for a flexible and straightforward view definition facility. In fact, in an OO model the view update problem is closely related to the nature of view instances $[7,8,10]$ because of the existence of object identifiers (object identity). In an object-preserving model, an update on a view instance is automatically propagated to the base instance, whereas in an object-generating model, it is necessary to hold the correspondence between every generated view instance and its base instances, and some updates are not possible. Obviously, object-generating views are necessary for supporting the simulation of the complete range of database schema changes. Bearing in mind the view functionality that we have chosen, our proposal includes object-preserving views and so, a view can add only operations, derived attributes and derived relationships. 


\section{The ODMG 3.0 Object Model}

In this section we summarize the basic concepts of the ODMG 3.0 object model. In the following section, we describe how views can be included in this object model. A detailed description of the ODMG 3.0 standard, including the Object Definition Language (ODL) and the Object Query Language (OQL), can be found in [5].

Objects and Literals. The basic modeling primitives are the object and the literal. An object has a state, defined by the values of its properties, and a behavior, defined by the set of operations that can be invoked on the object. Each object has a unique object identifier which does not change for the entire object lifetime, even if its attribute values or relationships do. A literal does not have identifier and its value cannot change. Both objects and literals are categorized by their types.

Literal Types and Object Types. The ODMG object model defines literal types and object types. Atomic object types are user-defined types, defined by type specifications. A type specification may be either an interface, defining only the abstract behavior of an object type, or a class, defining both abstract behavior and abstract state of an object type. By contrast, a literal definition defines only the abstract state of a literal type.

Throughout this paper we use as running example a database schema representing employees' information managed by a company (i.e. a university); this schema could include the Person interface and Department, Employee and Professor classes, whose ODL declarations are shown in Fig.s 1 and 2.

\begin{tabular}{|l|l|}
\hline $\begin{array}{l}\text { interface Person \{ } \\
\text { attribute string name; }\end{array}$ & $\begin{array}{l}\text { class Department } \\
\text { (extent departments ) } \\
\text { attribute string address; }\end{array}$ \\
$\begin{array}{l}\text { attribute enum gender }\{\text { male, female }\} ; \\
\text { attribute set<string> phones; }\end{array}$ & $\begin{array}{l}\text { attribute string name; } \\
\text { attribute string officeld; } \\
\text { unsigned short age ( ); } \\
\text { relationship set<Employee> workers }\end{array}$ \\
& inverse Employee::works_for; \\
\hline
\end{tabular}

Figure 1. ODL Specifications of Person and Department types

A type specification defines the external characteristics of the object type-visible to users of the type- as the operations that can be invoked on its instances, and the properties whose values can be accessed. The ODMG object model defines two kinds of properties: attributes and relationships. As is shown in the example, attribute declarations may appear in either a class or an interface specification. In a class, attribute declarations define the abstract state of its instances, whereas in an interface defines only abstract behavior. Relationships are defined between two object types -nary relationships are not supported- and may be one-to-one, one-to-many or many-to-many, depending on how many instances of each type participate in the relationship. The definition of a one-to-many relationship between Department and Employee types is shown in Fig.s 1 and 2: each employee is associated to a single department via works_for traversal path, and each department is connected with a set of employees via the workers traversal path. A relationship declaration in a class defines the abstract state for storing and the set of operations for accessing the relationship, 
whereas within an interface it defines only the operations of the relationship, not the state.

Subtyping and Inheritance of State and Behavior. The ODMG object model defines two subtype/supertype relationships: $I S-A$ and $E X T E N D S$. The $I S-A$ relationship defines the inheritance of behavior between object types. Only interfaces and classes can inherit from interfaces. The object model supports multiple inheritance of object behavior. On the other hand, the EXTENDS relationship defines the inheritance of state and behavior between classes and it is a single inheritance relationship. In our running example, the class Employee IS-A Person and the class Professor EXTENDS Employee, as is shown in Fig. 2.

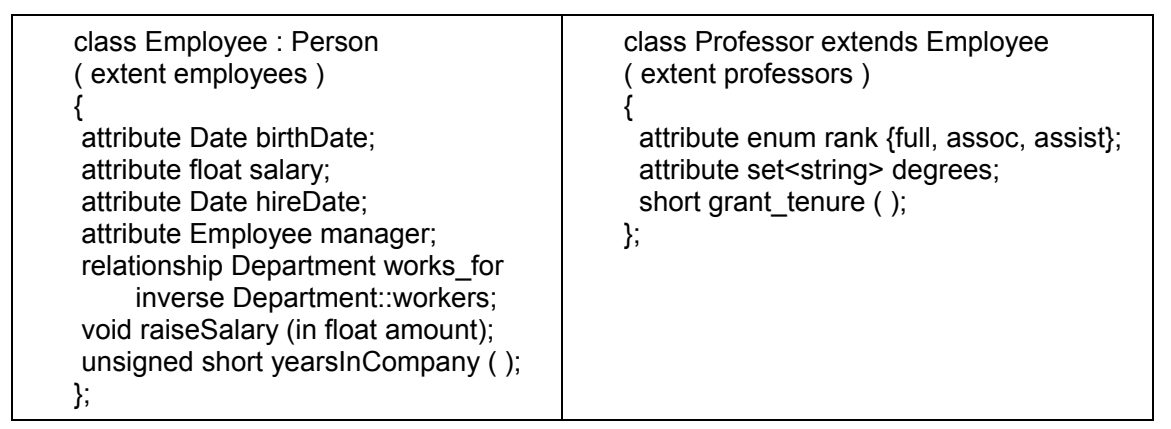

Figure 2. ODL Specifications of Employee and Professor classes

The extent of a type is the set of all instances of the type within a particular object database management system. Thus, every instance of the Professor class is a member of the extent of Professor, named professors in the corresponding ODL specification.

The subtype/supertype relationships (ISA and EXTENDS) involve polymorphism: where an object of the supertype can be used, an object of the subtype can be used instead. The extent of a subtype is a subset of the extent of its supertype; in the example in Fig. 2, professors is a subset of employees. An object's most specific type is the type that describes all features of the instance.

\section{Introducing Views in the ODMG Object Model and ODL}

To support a new concept in the ODMG object model, it is necessary to make some changes or extensions in the rest of its components. In our case, the inclusion of the view concept has meant extending the ODL and the bindings to the programming languages. However, it has not been necessary to make any modification in the query language, OQL, because views have the same nature as the rest of ODMG data types. In this section we describe how the object model and the ODL have been extended to include views.

We propose to introduce views as a new kind of data type and thus views could be used in the definition of a database schema. Just like classes and interfaces, views define the abstract state and/or the abstract behavior of an object type. Both the behavior and state of a view can include a portion of the behavior and state of its base class, and can also add new operations, derived attributes and derived relationships. The extent of the view, a subset of the extent of its 
base class, is defined by an OQL predicate which we denominate view invariant ${ }^{1}$.

\subsection{Definition of Views}

The structure of a view specification is shown in the following ODL code defining $V$ as a view of the $C$ base class:

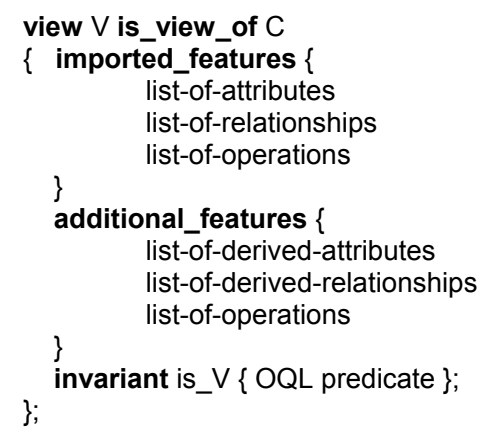

Thus, a view definition may include the following:

1. The view identifier

2. A specification of the base class from which the view has been derived. A view can have only one base class, because the view instances preserve the identity of the stored objects. Nevertheless, we will show later how it is possible to express views derived by a join operation.

3. A list containing the base class features which have been included in the view.

4. An abstract specification of the additional features, when the view adds new operations, derived attributes or derived relationships.

5. The view invariant, named in the form is_<view-name $>$.

We note that imported_features, additional_features and invariant declarations are optional but at least one of them has to be specified. The schema of our running example could include a view of the Employee class, named SeniorEmployee, the definition of which is given in Fig. 3.

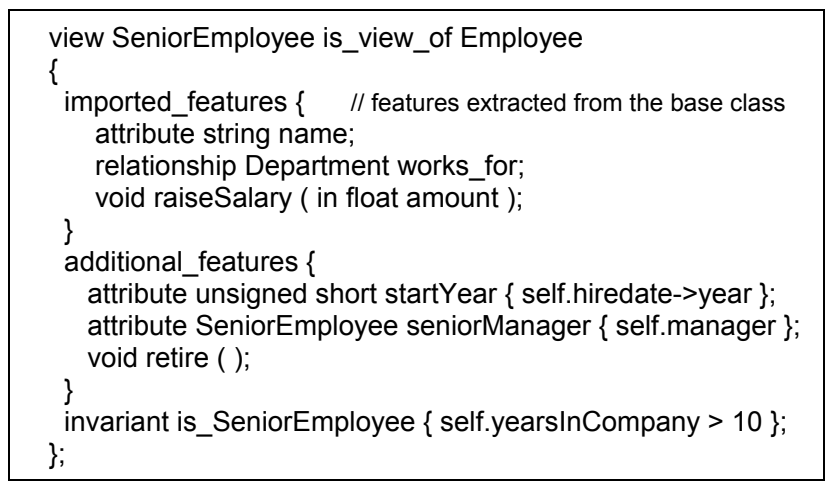

Figure 3. ODL specification of the SeniorEmployee type, as a view of the Employee class

\footnotetext{
${ }^{1}$ We use the view invariant term because it corresponds to the concept of class invariant. We think that this term is more appropriate than the usually used view query term because the ODMG object model considers that classes have only an intensional -but not extensional- nature, and thus the queries are applied on object collections.
} 


\subsection{Nature of the View Instances and Placement of Views}

Just like interfaces, views are types that are not directly instantiable. An instance of an interface is always a direct instance of a class that implements the interface. In a similar way, a view instance is a direct instance of its base class. In the previous example, SeniorEmployee is a view of Employee, so SeniorEmployee instances take their identity from the corresponding Employee instances. In this way, the objects stored in the database are not affected by the introduction of a new view in the schema. It is just that a stored object can act as an instance of a view. For example, an instance of the Employee class satisfying the is_SeniorEmployee predicate could act as an instance of the SeniorEmployee view. Therefore, from the point of view of the type compatibility, a view is compatible with its base type (i.e. an object whose type is SeniorEmployee may be connected to an Employee type variable).

With regard to the problem of inserting views in the schema, we use the view derivation relationship presented in $[1,7]$. We refer to this relationship as IS-VIEW. The alternative choice is to integrate every view in the class hierarchy as a subclass of its base class $[9,10]$, but this strategy requires a data model with multiple classification because a view instance would also be an instance of its base class. Moreover, the extent of a view is a subset of the extent of its base class, but the set of features of a view does not have to be a subset of the features of its base class.

\subsection{Features of a View}

Importation of Features by a View. A view may import attributes, relationships and operations already defined in the base class, albeit directly or inherited from any supertype. In the example, SeniorEmployee imports the name attribute, the works_for relationship and the raiseSalary operation.

Given a relationship $R$ between two classes $C 1$ and $C 2$, a view derived from $C 1$ or $C 2$ can import $R$. For example, SeniorEmployee imports the one-to-many relationship defined by the works for and workers traversal paths. When a view imports a relationship, the users of the view can only access a subset of the instances of the original relationship. The works_for relationship imported by SeniorEmployee consists in pairs <set of Employee, Department $>$ where the employees are instances of the view -and not all the objects of Employee. Thus, the original relationship between Employee and the Department class is viewed as a relationship between SeniorEmployee and Department. It is worth noting that the inverse of workers in Department is still works_for in Employee.

It should be observed that only the features imported from the base class are accessible in the view because the ISVIEW relationship is not an inheritance relationship. Thus, SeniorEmployee does not include salary, hireDate, manager or yearsInCompany features of Employee, nor other features of the supertype Person.

Definition of New Features in a View. A view may add new features. In the example, SeniorEmployee adds a new operation named retire, and two new attributes, startYear and seniorManager. Every new attribute introduced in a view 
always has to be a derived attribute whose value we know how to obtain. Thus, startYear is derived from the hireDate attribute by calculating the year of joining company, and seniorManager is derived from the manager attribute by returning either the manager, if he or she is also a senior employee, or the null value. OQL can be used to express the derivation expressions of new operations and derived attributes.

A view may include nested properties of the base class. That is, a view can add an attribute defined by a path expression starting from the base object. In our example, the SeniorEmployee view could include an attribute workOffice whose definition would be the following:

attribute string workOffice $\{$ self.works_for.officeld $\}$;

In addition to operations and derived attributes, a view may add new derived relationships. Given a relationship $R$ between two classes $C 1$ and $C 2$, and letting $V 1$ and $V 2$ be two views derived from $C 1$ and $C 2$ respectively, then it is possible to define a new relationship $R^{\prime}$ between $V 1$ and $V 2$ if and only if $R^{\prime}$ is derived from the original relationship $R$. A derived relationship $R$ ' defines a subset of the possible logical connections between the objects participating in the original relationship $R$. In our example, the relationship defined between the Employee and Department classes could be used to derive a new relationship between SeniorEmployee and a view of Department. A derived relationship, in a similar way to derived attributes, does not mean the addition of any new field to the view instances.

\subsection{The Invariant of a View}

The view invariant is an OQL predicate expressing the constraints that an instance of the base class must satisfy in order to act as a view instance. In the SeniorEmployee view, the invariant expresses that a senior employee is an employee who has worked at the company for more than ten years, so the extent of the SeniorEmployee view is the subset of employees whose yearsInCompany operation returns a value greater than 10. Just like an operation, a view invariant is always applied on an instance of the base class (the base object), and thus a path expression within the OQL predicate may start from either a variable connected to an object or from the reserved word self denoting the base object. An example of each kind of path expression appears in the FloralPerson view, shown below, whose view invariant contains f.name and self.name expressions. If a path expression does not start from either a variable or self, the base object is assumed.

As we mentioned before, a view definition may not to include any invariant declaration. In that case a true is assumed and therefore all the instances of the base class also belong to the view.

Joins in the View Invariant. It is worth noting that the view invariant specification allows us to express explicit joins, and thus the view can involve some more classes in addition to the base class. In the ODMG standard the explicit join is illustrated by an example selecting "the people who bear the name of a flower". In our approach, we could define the 
following view derived from the Person class:

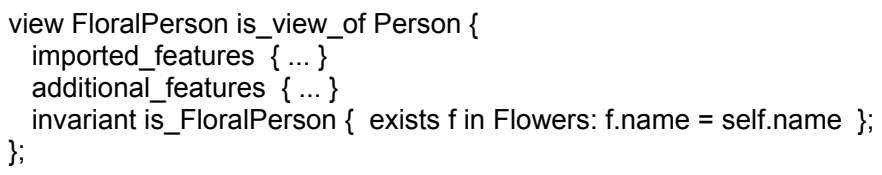

where Flowers denotes a collection of instances of the Flower class. In this view, the invariant includes an explicit join expressing that the name of a flower must be equal to the name of the person.

Views with Features Imported from More than One Class. Since a view has only one base class, all features imported by the view belong to its base class. Therefore, the FloralPerson view could only import features from the Person class but not from the Flower class. This restriction is originated by our choice of a view semantics with objectpreservation. In any case, operations or derived attributes could be used in order to include in a view operations and attributes belonging to those types involved in the view invariant and different to its base class. In the example, the FloralPerson view could include a scientificName operation that, given a Person instance belonging to the FloralPerson view, will return the value of scientificName attribute in the corresponding instance of the Flower class of the same name.

\subsection{Views and Inheritance}

A view defines the state and behavior of its instances. Both of them are always extracted from the base class and so they do not belong to the view itself. In addition, just like an interface, a view is not instantiable. Therefore, according to the nature of views, it does not make any sense to define subtype/supertype relationships between classes (or interfaces) and views. However, a subtype/supertype relationship between views could be defined. Hence, we have considered the view inheritance relationship introduced in [7], which has been revised and adapted to the ODMG context. This inheritance relationship has been named $V_{-} I S A$ and is orthogonal to the view derivation relationship. The whole set of possible supertype/subtype relationships in ODMG is summarized in Table 1 . It is worth noting that the $V$ ISA relationship has a different semantics to the $I S A$ relationship, although there are some common properties such as multiple inheritance and a polymorphic nature.

Table 1. Subtype/supertype relationships in ODMG extended with views

\begin{tabular}{|c|c|c|c|}
\hline \multirow{2}{*}{ Subtype } & \multicolumn{3}{c|}{ Supertype } \\
\cline { 2 - 4 } & interface & class & view \\
\hline interface & IS-A & - & - \\
\hline class & IS-A & EXTENDS & - \\
\hline view & - & - & V_IS-A \\
\hline
\end{tabular}

The following figure, which is an adaptation of Fig. 2-2 in [5], shows the possible relationships between views, interfaces and classes in the ODMG object model extended with views and it indicates whether multiple or single inheritance is allowed; it also includes the view derivation relationship. 


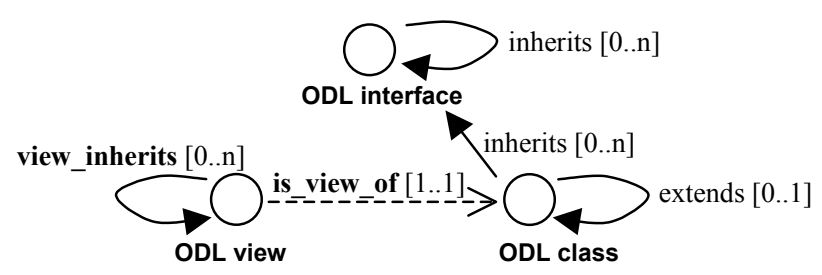

Figure 4. Class-Interface-View Relationships

In order to describe the $V_{-} I S A$ relationship, we use the ODL type hierarchy shown in Fig. 5 and based on our running example, where the SeniorEmployee view inherits from ReliablePerson.

The $V_{-} I S A$ relationship is defined as follows. Let a view be a subtype (a subview) of another view (the superview), then i) the subview inherits all the features of the superview and the subview may add new features (that are always either imported from its own base class or new operations and derived properties), ii) the $V_{-} I S A$ relationship involves polymorphism: an instance of the subview can act as a superview instance, and iii) the instances of the subview are those instances of its base class that satisfy both the subview and superview invariants; therefore, the subview instances are a subset of the superview instances. To ensure this, the following constraints have to be satisfied: i) the base class of the subview must be either the base class of the superview or a subclass of the base class of the superview, and ii) the invariant of the view must be added to the supertype invariant.

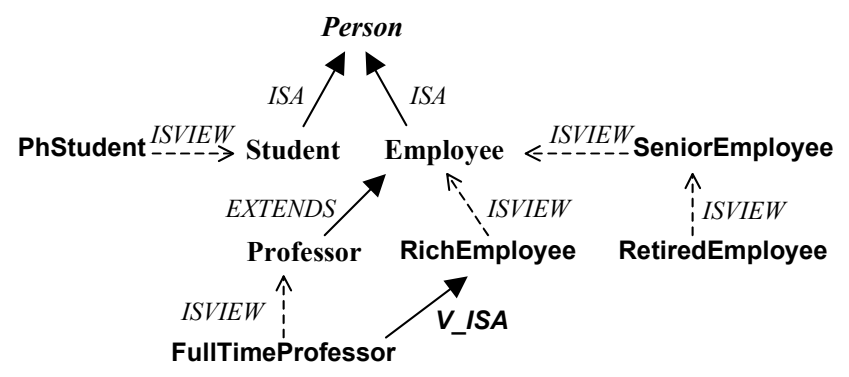

Figure 5. An ODL type hierarchy including views

In our example, the FullTimeProfessor view has been declared as a subview of the RichEmployee view. The FullTimeProfessor view could also inherit from SeniorEmployee or RetiredEmployee views. However, it could not be defined as a subview of the PhStudent view because this originates a situation in which multiple classification is required: supposing that FullTimeProfessor inherits from PhStudent, then every FullTimeProfessor instance -a Professor instance- is also a PhStudent instance, that is a Student instance.

It is worth noting that we could define a new view of Employee, named RichSeniorEmployee, which is a subview of both RichEmployee and SeniorEmployee views.

Finally, we can point out the following properties of our proposed view concept. Let $V C$ be a view whose base class is $C$, then:

- The extent of $V C$ is a subset of the extent of $C$. The instances of $V C$ are the instances of $C$ that satisfy the view in- 
variant. Obviously, as the instances of any subtype of $C$ belong to the extent of $C$, they will also be instances of $V C$ if they satisfy its invariant.

- The features of $V C$ are those declared in its definition. These can be features imported from $C$ or additional features (operations, derived relationships and derived attributes). $V C$ does not implicitly inherit other features from $C$ or from the supertypes of $C$.

- The $V C$ type is compatible with $C$, that is, an object of type $V C$ may be assigned to a variable of type $C$. The assignment of an object of type $C$ to a variable of type $V C$ requires a dynamic check of the view invariant for the assigned object.

- The ODMG supports parametrized collection objects such as $S e t<T>$ or $B a g<T>$ which implement the Collection $<T>$ interface. Types Collection $<V C>$ and Collection $<C>$ are compatible with each other. The assignment between them is always allowed. Since $V C$ instances are also $C$ instances, the assignment of an object of type $C o l l e c-$ tion $<V C>$ to a variable $c c$ of type Collection $<C>$ does not suppose any problem, whereas the assignment of an object of type Collection $<C>$ to a variable $v c c$ of type Collection $<V C>$ requires the dynamic check of the view invariant for all objects in the assigned collection because $v c c$ must contain only the objects that satisfy the invariant. The semantic of this assignment is discussed later in Sections 5.6 and 6.

Optionally, if $V C$ is declared as a subtype of another view $V$, then:

- $V$ must be either a view of $C$ or a view of any (direct or indirect) supertype of $C$.

- An inheritance relationship between views is established ( $\left.V_{-} I S A\right)$.

- $V C$ inherits all the features of $V$, and the invariant of $V C$ includes the $V$ invariant.

\section{Mapping Views Into $\mathrm{C}++$ Classes}

The ODMG standard defines the binding between the object model and the ODL and C++, Smalltalk and Java programming languages. Therefore, our proposal must extend these bindings in order to map the view concepts introduced in the object model into specific constructs of these OO programming languages. This mapping has to solve issues such as: how to place views within the class hierarchy, how to ensure that the user of the view only accesses a portion of the information, and how to solve compatibility problems between types. In this section, we describe the architecture of an OO database system supporting views and the mapping of views into $\mathrm{C}++$ classes.

\subsection{Database System Architecture}

Fig. 6 shows a global view of the architecture of a database system supporting the proposed view model. This figure is a new version of figure 5-1 in the specification of ODMG [5]. It is modified in order to manage ODL specifications with views, and to map views into $\mathrm{C}++$ classes. 


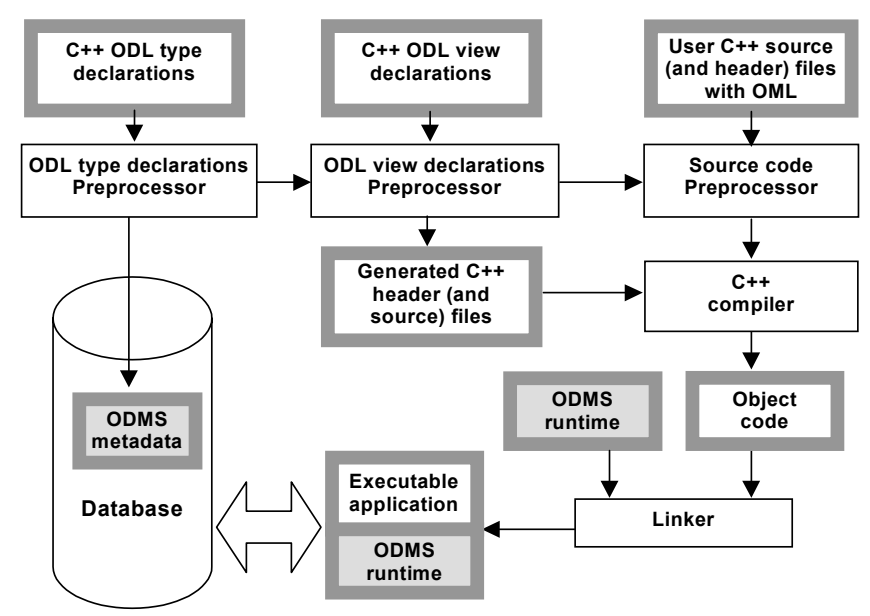

Figure 6. ODMG DBMS architecture including views

The database schema is defined by ODL declarations and the database applications are written in a high level programming language (we assume $\mathrm{C}++$ ). As Fig. 6 shows, we separate the standard ODL type declarations from the view declarations because views are derived once the conceptual schema has been established. Therefore, the declaration of classes and interfaces in the conceptual schema is not dependent on the set of defined views. The database conceptual schema is not modified when a new view is defined.

Fig. 6 includes two preprocessors related to views: the ODL view declarations preprocessor, and the Source code preprocessor. The first one translates view declarations into $\mathrm{C}++$ class definitions. A class implementing a view could be implemented either within the database application code (if the proper access privileges on the base class have been granted to the programmer) or by the database designer-the class here being imported into the application. The ODL view declarations preprocessor receives the result of the ODL type declarations preprocessor as input, because it needs to know the class declarations from which the views are derived. The ODL type declarations preprocessor included in the standard also has to be modified to perform the mapping into $\mathrm{C}++$ classes in a slightly different way on account of the problem explained below in Section 5.2. The output of the ODL view declarations preprocessor is a set of header and source files to be included in the $\mathrm{C}++$ application.

The translation of views into class declarations must be completed by also preprocessing the application source code. This step ensures that an application only uses the types on which it holds the proper access privileges. Finally, the compiler receives the generated $\mathrm{C}++$ header and source files with the structure of the ODL types (including views), and the application source code.

\subsection{Mapping ODL Classes}

The ODMG standard establishes a syntactic equivalence between attributes of ODL types and data member of C++ classes, so an ODL class attribute is mapped into a public data member of the corresponding $\mathrm{C}++$ class. Here, we introduce a modification to the standard because, as we will see later, it is necessary for a subclass to be able to modify the 
$\mathrm{C}++$ data member export status. Let $B$ be a subclass of $A, \mathrm{C}++$ allows $B$ to redeclare any function member by changing its export status, but it does not allow this kind of redeclaration for data members.

Therefore, in our model each ODL class attribute is mapped into a protected data member and two public function members ${ }^{2}$, one that returns the attribute value and another that allows the direct modification of the attribute (get and set methods). To increase efficiency, the compiler may detect calls to public methods that only return the value of an attribute and may handle them just like a direct access to such an attribute. Fig. 7 illustrates how ODL class declarations are translated into $\mathrm{C}++$ class definitions. It should be noticed that all methods are declared as virtual, and since repeated inheritance will arise in the $\mathrm{C}++$ class hierarchies obtained, virtual inheritance is used.

\begin{tabular}{|c|c|}
\hline ODL Classes & $\mathrm{C}++$ Classes \\
\hline $\begin{array}{l}\text { class } \mathrm{A} \\
\left\{\begin{array}{l}\text { readonly } \\
\text { attribute long } \mathrm{q} ;\end{array}\right.\end{array}$ & $\begin{array}{l}\text { Class A: public d_object }\{ \\
\text { public: } \\
\text { virtual d_Long q (void); } \\
\text { protected: } \\
\text { d_Long_q; } \\
\text { \}; }\end{array}$ \\
\hline 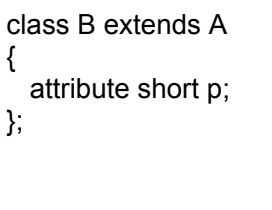 & $\begin{array}{l}\text { Class B: public virtual A }\{ \\
\text { public: } \\
\text { virtual void p(d_Short value); } \\
\text { virtual d_Short p (void); } \\
\text { protected: } \\
\text { d_Short_p; } \\
\text { \}; }\end{array}$ \\
\hline
\end{tabular}

Figure 7. An example of the mapping of ODL classes into C++ classes

\subsection{Mapping ODL Views}

In our work, one of the main issues that we have tackled is the translation of views into classes in the chosen programming language. In particular, it is necessary to find out how to translate the $I S$-VIEW relationship into $\mathrm{C}++$ constructs. Bearing in mind the aforementioned problems arising when integrating a view as a subclass of its base class (primarily, multiple classification is needed), we have devised the following mapping.

A view $V C$ derived from the base class $C$ is mapped into a $\mathrm{C}++$ abstract class with the same name. In the $\mathrm{C}++$ hierarchy, this class is a new superclass of the class $C$ and a subclass of all the classes corresponding to the supertypes of $C$ within the ODL type hierarchy. That is, $V C$ is placed between $C$ and its superclasses. Obviously, this approach needs multiple inheritance because both $C$ and $V C$ classes may have one or more superclasses. From now on, we refer to the $\mathrm{C}++$ abstract class corresponding to an ODL view as class-view.

Since a class-view is an abstract class, it includes a set of abstract methods and is not instantiable. Therefore, the extent of a class-view consists of direct instances of its base class or its subclasses, which are objects stored on database. Fig. 8 shows a graphic representation of an example of mapping of ODL type declarations into C++ classes. In this example, we have derived three views from the class $B$ introduced in Section 5.2: $V 1$ and $V 2$ are derived from the class

\footnotetext{
${ }^{2}$ In the $\mathrm{C}++$ context, we use the more general terms method and attribute instead of function member and data member, respectively.
} 
$B$, and $V 3$ is a view of $V 2$. The "*" symbol denotes an abstract class, whereas the "+" symbol denotes an effective (nonabstract) class. Throughout this section, we use as running example the hierarchies presented in Fig. 8.

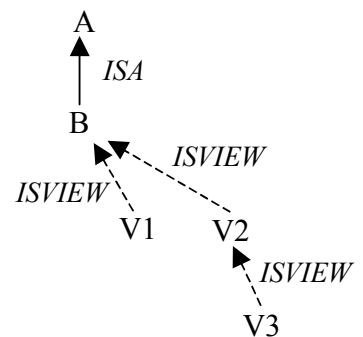

(a) ODL type hierarchy

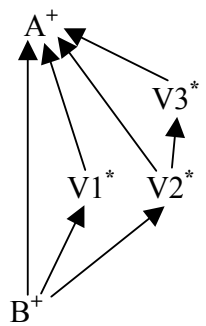

(b) C++ class hierarchy

Figure 8. An ODL type hierarchy and its mapping into $\mathrm{C}++$

The definition of views $V 1$ and $V 2$ requires the declaration of the $\mathrm{C}++$ class $B$ to be modified in order to include the two views as superclasses:

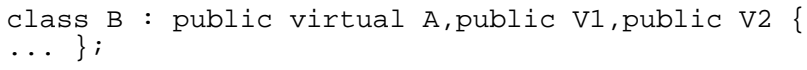

Bearing in mind that the ODL class $B$ has the $q$ attribute, inherited from $A$, and the $p$ attribute, which is directly introduced in $B$ (see Fig. 7), then we can distinguish several mapping cases according to whether the attributes $p$ and $q$ are imported or not by the $V 1$ view, and whether the view introduces new features or not. Although only attributes have been considered, the discussion for each case is also suitable for operations because the mapping of the ODL attributes involves operations.

\section{- $V 1$ imports the $p$ attribute from $B$}

The $V 1$ class-view has to declare the methods corresponding to this attribute as public and abstract. The subclass $B$ makes them effective, without any further modifications.

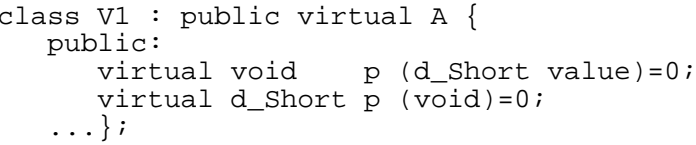

\section{- $V 1$ does not import the $p$ attribute}

The $V 1$ class-view does not have to include any method concerning $p$.

\section{- $V 1$ imports the $q$ attribute introduced in $A$}

The $V 1$ class-view already inherits the $q$ attribute from $A$, so it should not include any method related to $q$. There may exist one or more views derived from $B$ and each corresponding class-view establishes an inheritance path between $B$ and $A$. Virtual inheritance is applied between each class-view and $A$ so that the attributes of $A$ are not replicated in $B$. 


\section{- $V 1$ does not import the $q$ attribute introduced in $A$}

$\mathrm{C}++$ public methods corresponding to the attribute $q$ have to be redefined in the $V 1$ class-view. Their export status must be changed from public to protected in order to hide them from the clients, and the method body must be replaced by an empty block. Since such methods are inherited as protected by $B$, they must be redeclared as public in

$B$.

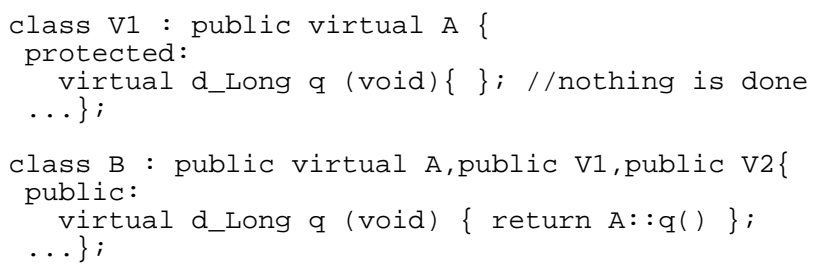

\section{- $V 1$ introduces a new operation or a new derived attribute $t$}

Since a view can only add operations and derived attributes, only methods can be added. The $V 1$ class-view declares a new virtual method $t$. Since the $B$ class inherits this method, $B$ has to hide it from its clients and subclasses and therefore, the $t$ method is redeclared as private with the same implementation.

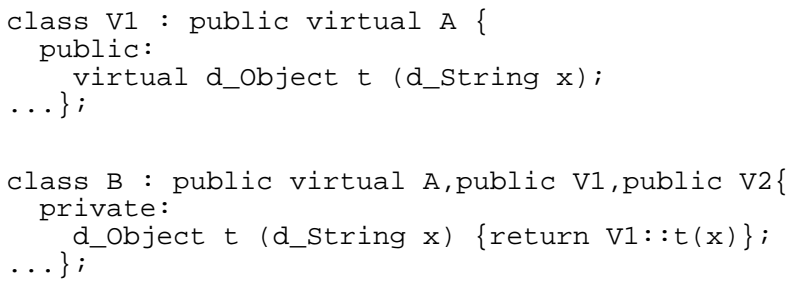

With regard to relationships, we have to distinguish between importation and addition. In ODMG, a relationship is mapped into an attribute (used to refer to the other end of the relationship) plus several methods on the relationship target classes. These methods form and drop members from the relationship, provide access and manage the required referential integrity constraints. Therefore, the mapping of an imported relationship is the same as the mapping described for imported attributes and operations. When a derived relationship is added within a view, each relationship target view-class has to include a method which returns the other end plus the methods managing the relationship and which delegate their tasks to the corresponding methods (generated from the ODMG mapping) in the base class.

The existence of the $V 2$ view does not affect the mapping established for each case, because we have considered virtual inheritance, so the $B$ class would share all attributes and methods inherited from each class-view. We have verified that ambiguous situations do not arise. Moreover, these mapping rules can be applied regardless of base type of view be it a class or a view- within the ODL declaration. In the running example, $V 3$ is a view of $V 2$, then $V 3$ is mapped into a new superclass of the $V 2$ class-view and a subclass of the superclass of $V 2$-the $A$ class.

\subsection{The View Invariant}

In an ODL view specification, the view invariant is an OQL predicate that expresses the constraints which must be 
satisfied by a view instance; let the view $V$ be derived from the base class $C$, then every instance of $C$ satisfying the view invariant of $V$ is also an instance of $V$. Therefore, the view invariant defines the extent of a view as a subset of the extent of its base class.

According to the specialization semantic of the $I S$ - $A$ relationship, every instance of a class $C$ can act as an instance of any superclass of $C$, thus the extent of a class $C$ is a subset of the extent of any superclass of $C$-the extent of $C$ is made up of the direct instances of $C$ and the instances of its subclasses. However, our approach for mapping views into $\mathrm{C}++$ classes does not follow this semantics strictly because a class-view is a superclass of the base class of that view, and so an instance of the base class can only act as an instance of the class-view if it satisfies the invariant. Nevertheless, our solution works since the class-view is an abstract class which has a single subclass, the base class of that view, and so all the instances of the class-view will also be instances of the single subclass. An entity (variable, parameter) whose type is a view, could only reference instances of the base class, with the only condition that it will be necessary to check the view invariant whenever an instance of the class attempts to act as an instance of a class-view (in a similar way as a type cast). So, we think that the object-oriented paradigm is preserved.

Given the following variable declarations

ob: B; ov1: V1

where $V I$ and $B$ are the classes of the example showed in Fig. 8, the assignment

$$
\mathrm{ov} 1=\mathrm{ob}
$$

would be legal, although at runtime the object connected to $o b$ will only be connected to $o v 1$ if that object satisfies the view invariant of the $V 1$ view. For example, an entity whose static type is SeniorEmployee, can only be connected to an Employee instance satisfying the invariant of the SeniorEmployee view.

In the $\mathrm{C}++$ binding, a view invariant is mapped into several $\mathrm{C}++$ methods. First, the $d$ _Object class, whose purpose is to enable a definition of a persistent-capable class, is extended with a new method named check predicate whose signature is

d_Boolean check_predicate(const char* OQL_predicate);

This method has the OQL predicate expressing the view invariant as input and returns true or false depending on whether the predicate is satisfied or not by the target object. Secondly, every class-view will include an automatically generated method, named like the view invariant in the ODL declaration (i.e. $i_{-}<v i e w-n a m e>$ ), whose purpose is to check whether the view invariant predicate is satisfied or not. This method has no arguments and invokes the check predicate method and provides it with the view invariant predicate as argument. Finally, each $\mathrm{C}++$ class will include a non-virtual method named view_invariant whose purpose is either to invoke the $i_{-}<$view-name $>$method, in the case of a class-view, or to return true, in the case of a $\mathrm{C}++$ class corresponding to an ODL class or interface. In this 
way, methods which need to check whether a stored object belongs to a given view, perform the checking through a chain of three invocations: the view_invariant method is invoked first, and which in turn invokes the method is_<viewname>, which finally invokes the check_predicate method.

The implementation of the view invariant through two methods (view_invariant and is_<view-name $>$ ), instead of through a single method, is due to two facts: i) a class can have several views, and ii) some classes, such as iterators or collections, managing instances of any view, need to apply a generic method instead of an is_<view-name $>$ specific method to check the view invariant.

Fig. 9 shows the ODL definition of the $V 1$ view introduced in our running example, and the corresponding classview in $\mathrm{C}++$. The $B$ class inherits the methods $i s_{-} v 1, i s \_v 2$ and $i s \_v 3$ from the superclasses $V 1, V 2$ and $V 3$, respectively, and has a view_invariant method returning true. It is worth noting this method is not declared as virtual because the dynamic type of an entity whose static type is a view will always be the base class of the view or some subclass. In our example, given an entity ov 1 whose static type is $V 1$, the dynamic type of $o v 1$ will be $B$ or some subclass of $B$. By defining view_invariant as a non-virtual method, the message ovl->view_invariant() will always check the invariant of $V 1$, instead of returning true.

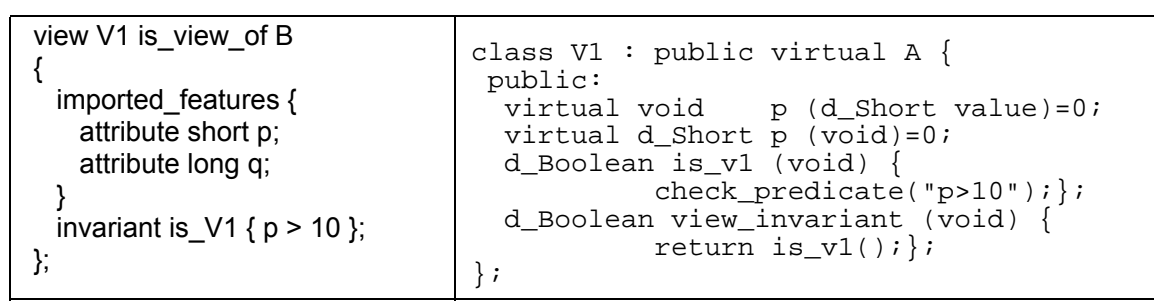

Figure 9. ODL definition of the $\mathrm{V} 1$ view and the mapping into a $\mathrm{C}++$ class

For example, given the method insert_element (const $T$ \&element) belonging to the template class $d \_$Set $<T>$, and supposing the instantiation $d$ _Set $<$ SeniorEmployee $>$, then the invocation of the message element->view_invariant() during the execution of the insert_element method will provoke the checking of the invariant of the SeniorEmployee view on the object connected to the element parameter. Management of collections is described in Sections 5.6 and 6.

A view $V 3$, whose base type is another view $V 2$, will include the invariant of $V 2$ :

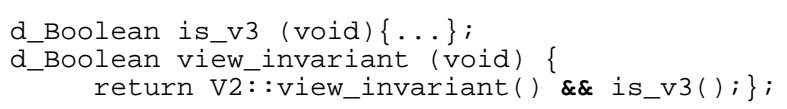

In the same way, if a view $V 3$ is declared as a descendant of another view $V 2$, then the invariant of $V 3$ is and-ed to the invariant of $V 2$. Moreover, if both relationships are given at the same time, i.e. if a view $V$ is derived from another view and is a subtype of a third view, then all invariants are and-ed within the view invariant of $V$ class-view.

\subsection{View Access Control}

A view mechanism facilitating the definition of external schemas must apply two controls aimed at constraining users to 
access to the portion of authorized information: i) the user application can only use the types (classes, interfaces and views) included in the external schema, and ii) an entity whose type is a view can only reference an instance satisfying the view invariant. The first control is performed at compilation time by the $\mathrm{C}++$ source code preprocessor which takes both the application source code and the external schema as input. The second control is performed at runtime by checking the view invariant in order to evaluate when a stored object can act as a view instance.

The ODMG $\mathrm{C}++$ binding defines a template class $d_{-}$Ref $<T>$ to allow references ${ }^{3}$ (smart pointers) between objects (either persistent or not). A variable of type $d_{-} \operatorname{Ref}<T>$ is a reference to an instance of type $T$, which can be dereferenced using * and $->$ operators, or assigned to another variable with the $=$ operator. According to the standard, if $D$ is a superclass of $C$ then a $d_{-} \operatorname{Re} f<C>$ can be assigned to a $d_{-} \operatorname{Re} f<D>$. This kind of assignment is possible through the $d_{-}$Ref_Any class, which is used as an intermediate step in the conversion between different instantiations of $d \_$Ref. We have modified the $d_{-} \operatorname{Ref}<T>$ class in order to check a view invariant. Thus, the source code preprocessor ensures that references to instances of a view $V$ are always performed through variables whose type is $d \_$Ref $<V>$, i.e. pointer variables are not used. The view invariant is checked each time that a variable whose type is a view is manipulated: i) it is initialized, ii) an object is assigned to it (the assignment operator has been modified in the $d_{-}$Ref template), and iii) a dereference is applied on it $\left(*\right.$ and $->$ operators have been also modified in $d_{-}$Ref).

If a view invariant is not satisfied, then a d_Error_RefInvalid exception is raised, indicating an attempted access to unauthorized information. In any case, the client code could avoid this runtime exception by checking the view invariant before executing the instruction that raises the exception; this checking could be performed by means of the invocation of the method that implements the view invariant predicate in the class-view. Some changes in the state of a view instance may make the view invariant not be satisfied in the new state. For this reason, the view invariant is also checked when a $d \_R e f<T>$ is de-referenced. This test adds a time overhead to the use of views which is proportional to the time required to execute the invariant. In any case, optimization techniques could be used to partially avoid this increment in the execution time.

\subsection{Mapping Collections}

Collections play an important role in object-oriented databases because users normally use them to access to the stored information. Now, it is possible to declare collections whose elements are of a view type, for instance $d \_$Set $<$SeniorEmployee $>$. Since the ODMG standard enforces the compatibility between a collection of a type $T$ and a collection of a supertype of $T$, a user will be able to access a collection containing instances of a class $C$ through a vari-

\footnotetext{
${ }^{3}$ ODMG specifies that "a reference in many aspects behaves like a $\mathrm{C}++$ pointer, but provides an additional mechanism that guarantees integrity in references to persistent objects".
} 
able declared as a collection of instances of a view derived from $C$. For example, a user who is allowed to access SeniorEmployee but not Employee, can obtain a reference to a collection $d_{-}$Set $<$Employee $>$, but through a $d \_$Set $<$SeniorEmployee $>$reference. It is necessary to ensure that the user can only see the employees satisfying the SeniorEmployee view invariant.

With regard to the implementation, now a collection needs to know if its elements are being considered as instances of some view. In other words, the behavior of the methods in the classes associated to collections $\left(d \_S e t, d \_B a g, d \_L i s t\right.$, $d_{-}$Array, $d_{-}$Varray, $d_{-}$Dictionary) and in the class of the iterators (d_Iterator) has to be modified to check, when necessary, the view invariant of the type of the collection. This modification only causes some changes in the generic implementation of the collections and iterator templates. These issues are explained in the next section.

\section{Accessing Stored Objects through Views}

According to the ANSI/SPARC three-level architecture, an external schema offers a vision of the database schema for a particular user or group of users. Therefore, all users associated to an external schema have to hold access privileges on all the types included in that schema. In this section we discuss the definition and use of external schemas defined by the view concept proposed for the ODMG. We pay attention to the issues related to the access from a programming language to the stored objects.

Most object-oriented view models proposed have considered the definition of external schemas [7,9]. In our approach, an external schema is defined by the derivation of views and the importation of types (classes, interfaces and views) from the conceptual database schema. As is well-known, an external schema must satisfy the closure property: for every type included in the external schema, all types used in the declaration of the attributes and operation parameters of that type must also belong to the schema. A detailed discussion about external schemas and the closure property can be found in [7].

Next, we consider a $\mathrm{C}++$ application accessing a database (a collection of objects belonging to classes included in the base schema) through an external schema and we analyze the issues related to the manipulation of the database from the application.

In ODMG, a logical database ${ }^{4}$ is an instance of the Database type. The Database interface includes the operations for manipulating the Database object, such as bind or lookup. The operation bind allows us to connect a name to a stored object. The objects in the database are accessible to an application through these named objects which are the entry point to the content of the database. The operation lookup allows retrieval of the stored object bound to a name

\footnotetext{
${ }^{4}$ ODMG specifies that "an OODBMS may manage one or more logical databases, each of which may be stored in one or more physical databases".
} 
supplied as a String argument. This operation returns the object as an instance of the root of the class hierarchy, that is the Object type. Therefore, an application retrieving objects from the database must perform a type cast in order to manipulate the objects extracted. For instance, let employees be the name of a set of instances of the Employee class, the following $\mathrm{C}++$ code shows how the employees object could be retrieved and assigned to a variable es, and the application of the cardinality method on this set. The first variable declaration means that es is a reference to a set of references to instances of the Employee class.

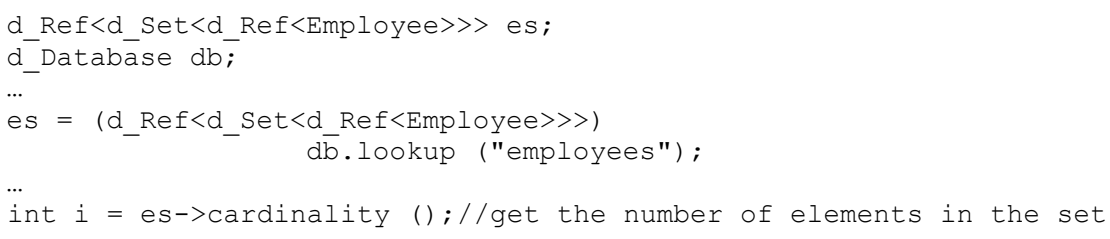

When a user is restricted to developing applications on an external schema, then he or she may only use the types included in that schema. Given an external schema including the SeniorEmployee view but not the Employee class, then the previous $\mathrm{C}++$ code would be written as follows:

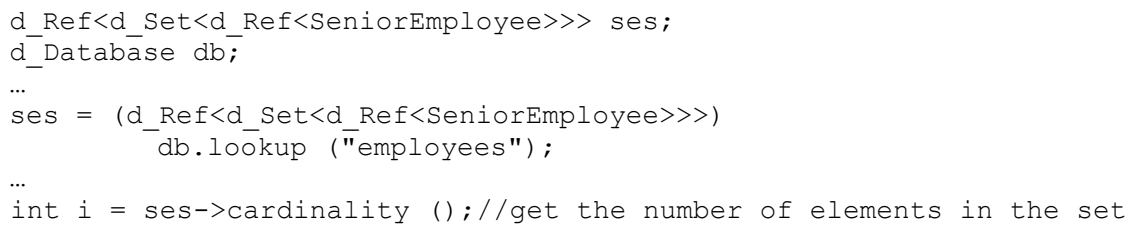

Now, the employees set is manipulated from the viewpoint provided by the SeniorEmployee view. Both es and ses variables reference the same set of Employee objects, but the static type of the actual parameter of the collection is different: Employee class and SeniorEmployee class-view respectively (as we explained above in Section 5.6, the types $d \_$Set $<$Employee $>$and $d$ _Set $<$ SeniorEmployee $>$ are compatible). Whereas all instances of the employees set can be accessed through a $d$ Set $<$ Employee $>$ reference, only the instances that satisfy the SeniorEmployee view invariant can be accessed through a $d$ _Set $<$ SeniorEmployee $>$ reference: the ses->cardinality() message returns the total number of senior employees, instead of the total number of employees returned by the es->cardinality() message.

Therefore, the checking of the view invariant should be included within the implementation code of the methods that manipulate collections (insert_element, remove_element, etc.) because, given a class-view $V$, a $d$ Collection $<V>$ reference allows access to a collection containing instances of the base class of $V$, but this reference should only allow access to instances of view $V$.

However, collections are not the only objects that can be stored in a object-oriented database. Objects of any type can be persistent (orthogonal persistence). For example, let us suppose that a named object john, referencing an instance of the Person class, is defined in the $d b$ database instance. A user restricted to using the SeniorEmployee view could retrieve the object john by executing the following assignment: 
Just as happens with a variable referencing a collection of view instances, the se variable allows access to the john object through the features of the view SeniorEmployee.

During the execution of a type casting, an exception is raised when the dynamic type of the object is not compatible with the static type of the variable. Furthermore, the assignment may now raise another exception if the assigned stored object violates the view invariant. In order to emphasize this difference, this type conversion may be called view casting, a particular case of type casting. It should be noticed that a reference whose static type is a view-class (for example, SeniorEmployee) can always be assigned to a variable whose type is the base class of that view (Employee).

\section{Conclusions and Future Work}

In this paper, we have presented a proposal for the inclusion of views in the ODMG standard. Firstly, we have discussed our main design decisions, assuming that $\mathrm{OO}$ views have the same functionality as relational views. We have presented the basic concepts of the ODMG object model and then we have described how the object model, the object definition language and the $\mathrm{C}++$ binding have been extended.

Views have been included in the object model in such a way that views are a new kind of ODMG data type and the $I S-V I E W$ relationship specifies the derivation of a view from its base class. The definition of a view specifies the list of features imported from its base class, the list of additional features and the view invariant (an OQL predicate defining the view extent). In a view model supporting capacity-augmenting views (that is, views that may add non-derived attributes), object restructuring is needed and object updates are more complicated. Hence, in our view model we have considered object-preserving views -that is, each view instance preserves the identity of its base instance- and views can only add derived attributes (and relationships) and new methods. Although in our approach, a view can only have a single base class, we have shown how several classes could be involved in a view declaration. Since views are a new kind of data type, it is not necessary to change the object query language, OQL.

Regarding the binding to the programming languages, we have described a $\mathrm{C}++$ binding. Each view is mapped into a $\mathrm{C}++$ abstract class which is placed, in the class hierarchy, between the $\mathrm{C}++$ base class and the superclass of this base class. With this implementation technique it is possible that a view instance preserves the identity of its base instance. We have discussed how the polymorphism is affected by integrating the view as a superclass of its base class: an instance of a base class can only act as a view instance if the view invariant is satisfied, in a similar way to a type cast. The process to generate $\mathrm{C}++$ code from ODL declarations has been modified by introducing two additional preprocessors in order to manage views in ODMG. Finally, we have described how to manage collections containing view instances. 
We believe that the $\mathrm{C}++$ binding obtained is practical. It can be implemented on a commercial OO database system because it does not impose hard restrictions. Besides, the object-oriented paradigm is preserved and the database programmer may write applications in the usual way. However, it is difficult to translate this binding into Smalltalk and Java, because our approach to mapping views into $\mathrm{C}++$ classes is based on multiple inheritance of classes and on some specific properties of $\mathrm{C}++$, such as the use of non-virtual methods (in the implementation of the view invariant). At present, we are working on the definition of Java and Smalltalk bindings. In the Java binding, the multiple inheritance of interfaces could be useful in order to include views in the class hierarchy.

Our future work also includes, i) to extend the ODMG metadata descriptions, and ii) to deal with the formalization of the view model in a similar way as is done in the formal view model presented in [7].

\section{References}

[1] Bertino, E.: A View Mechanism for Object-Oriented Databases. Proceedings of the 3rd International Conference on Extending Database Technology (EDBT), (1992) 136-151

[2] Bertino, E., Ferrari, E., Guerrini, G., Merlo, I.: Extending the ODMG Object Model with Time. Proc. of 12th European Conference on Object-Oriented Programming (ECOOP), Bruxelles (Belgium), July 20-24 LNCS, No.1445, Springer-Verlag (1998).

[3] Bertino, E., Guerrini, G.: Extending the ODMG Object Model with Composite Objects. Proceedings of ACM Object-Oriented Programming Systems, Languages and Applications (OOPSLA'98) Conference, Vancouver (Canada), Oct. 18-22, ACM Press (1998)

[4] Carey, M.J., DeWitt, D.J.: Of Objects and Databases: A Decade of Turmoil. Proceedings of the 22nd VLDB Conference, Bombay, India (1996) 1-12

[5] Cattell, R.G.G. et al.: The Object Database Standard: ODMG 3.0. Morgan Kaufmann Pub. (2000)

[6] García-Molina, J., Guerrini, G., Bertino, E., Catania, B.: Dimensions in the Design of an Object-Oriented View Mechanism. Proceedings of the 1st Spanish Conference on Databases JIDBD, A Coruña (1996) 119-129

[7] Guerrini, G., Bertino, E., Catania, B., García-Molina, J.: A Formal Model of Views for Object-Oriented Database Systems. Theory and Practice of Object Systems, Vol. 3(3), (1997) 157-183

[8] Kim, W., Kelley, W.: On View Support in Object-Oriented Database Systems. In: Kim, W. (ed.): Modern Database Systems. ACM Press (1995) 108-129

[9] Kuno, H.A., Rundensteiner, E.A.: The MultiView OODB View System: Design and Implementation. Theory and Practice of Object Systems, Vol. 2(3) (1996) 203-225

[10] Scholl, M.H., Laasch, C., Tresch, M.: Updatable Views in Object-Oriented Databases. Proceedings of the 2nd International Conference on Deductive and Object-Oriented Databases. LNCS, No. 566 (1991) 189-207

[11] Souza dos Santos, C.: Design and Implementation of Object-Oriented Views. Proceedings of the 6th International Conference DEXA. LCNS, No. 978 (1995) 91-102 